\title{
Towards data centric mobile security
}

\begin{abstract}
Recently the usage of mobile devices has increased rapidly and it will be growing even more as mobile devices functionality is at enhanced. So the security of mobile devices needs to be improved. This paper discusses various components and functions of mobile devices such as SMS, wireless and applications and describes possible vulnerabilities threatening them precisely. Malware threats are explained and their effects on mobile devices are studied. Finally, to protect all the vulnerabilities and prevent application and malware threats, datacentric security model is provided as a solution to ensure confidentiality, integrity and availability of data stored on mobile devices.
\end{abstract}

Keyword: Mobile devices; Data-centric; Malware threat 